\title{
Effects of Phased Rehabilitation Programs after Total Knee Arthroplasty: A Randomized Controlled Trial
}

\author{
Jing Chao ${ }^{1+}$, Lee Mingzhu ${ }^{2+}$, Gong Qi ${ }^{3}$, Bai Xuehua ${ }^{4}$, and Yang Peilei ${ }^{5}$ \\ 1 The Second Hospital of Hebei Medical University, Shijiazhuang, China; E-mail: \\ drjingchao1995@foxmail.com \\ 2 Chongqing Medical University, Chongqing, China; E-mail: 965233247@qq.com \\ 3 Wuhan Sports University, Wuhan, China; E-mail: 2445112113@qq.com \\ 4 Hebei Normal University Sports Rehabilitation Department, Shijiazhuang, China; E-mail: \\ 122177811@qq.com \\ 5 Peking University Health Science Center, Beijing, China; E-mail: 25441211@qq.com \\ * Correspondence: drjingchao1995@foxmail.com; Tel.: +86-0311-86213190 \\ $\dagger$ Jing Chao and Lee Mingzhu contributed equally to this article.
}

\begin{abstract}
This multi-center, single-blinded, randomized controlled study assessed the efficacy of phased exercise rehabilitation programs in patients who underwent a total knee arthroplasty (TKA) and investigated suitable exercise types, intensities, and frequencies for patients undergoing postoperative rehabilitation. Between January and March 2018, 494 patients who had undergone TKA were treated at two medical centers. Patients were randomized and allocated to the rehabilitation group ( $n=330$ ) or the control group $(n=164$; treated with postoperative care). The 100mm Visual Analogue Scale (VAS), Western Ontario and McMaster Universities Arthritis Index (WOMAC), range of motion, and Short-Form 36 (SF-36) scores were assessed and compared between the two groups. The average WOMAC score was $84.40 \pm 15.20$ in the rehabilitation group and $108.30 \pm 3.90$ in the control group $(p=0.009)$. The mean VAS score was $2.54 \pm 0.16$ in the rehabilitation group and $2.87 \pm 0.31$ in the control group $(p=0.024)$. Furthermore, the range of motion was $125.02 \pm 6.20$ in the rehabilitation group and $116.40 \pm 1.40$ in the control group $(p=0.017)$. The mean SF-36 score was $105.40 \pm 21.50$ in the rehabilitation group and $83.70 \pm 5.01$ in the control group $(p=0.043)$. This study suggests that the phase rehabilitation program could be more effective at improving pain, knee function, and quality of life than condition postoperative care after TKA.
\end{abstract}

Keywords: total knee arthroplasty; rehabilitation program; knee osteoarthritis; exercise rehabilitation; postoperative care

\section{Introduction}

Osteoarthritis is a chronic degenerative disease of the cartilage that mostly occurs in the knee joint [1]. The China Health and Retirement Survey reported that $8.1 \%$ of patients were diagnosed with knee osteoarthritis in 2015 [2], and that knee osteoarthritis is a common cause of knee pain and disability among middle-aged and older Chinese adults.

In general, treatment for patients with knee osteoarthritis focuses on relieving pain and improving daily knee function and movements. During the last decade, total knee arthroplasty (TKA) became the gold standard treatment for end-stage knee osteoarthritis [3], and most patients experienced successful long-term postoperative outcomes, reduced pain, and improvement in daily movement. Between 2003 and 2012, the incidence of TKA increased from 145.4 to 223.0 per 100,000 people in Australia [4]. Over 700,000 TKAs are performed each year in America to alleviate the pain and disabilities associated with knee osteoarthritis [5].

However, postoperative adverse events, including weakened quadriceps strength, decreased walking distance, and decreased stair climbing speed, are most common in patients who have undergone TKA [6]. Most guidelines suggest that physical rehabilitation after TKA is essential to 
improve functional outcomes and allow patients to safely return to daily activities $[7,8]$. However, current rehabilitation programs are controversial; the exercise types, intensities, frequencies, and timing of exercises are unclear, and there is no high-quality data to suggest that such programs are effective at improving short- and long-term outcomes [9].

Therefore, we aimed to assess the efficacy of phased rehabilitation programs in patients who have undergone TKA and to investigate suitable exercise types, intensities, and frequencies for patients undergoing postoperative rehabilitation. We found that phased rehabilitation programs were more effective than condition postoperative care to improve pain, knee function, and quality of life after TKA.

\section{Materials and Methods}

\subsection{Patient selection and ethical considerations}

This single-blinded multi-center, randomized controlled trial was approved by The Second Hospital of Hebei Medical University Ethics Committee (Registration No. 20180211) and the Chongqing Medical University Ethics Committee (Registration No. CMUE20180321), and also was registered at the Chinese Clinical Trial Registry (ChiCTR1800019208). This study was conducted in accordance with the Declaration of Helsinki for medical research involving human subjects.

In this study, we included patients who (1) were diagnosed with knee osteoarthritis and underwent TKA, (2) were between 60 and 70 years old, and (3) provided written informed consent for the clinical trial and agreed to comply with the protocol requirements of this study. We excluded patients who had (1) severe pain and swelling, (2) postoperative infections, (3) severe organ dysfunction; in particular, we excluded patients with cardiovascular diseases (class III or above according to the New York Heart Association classification), chronic kidney disease (level III or above according to their outcome conditions), or liver disease (according to a model of end-stage liver disease score of 20 or above), (4) severe mental illness, and (5) failed to provide informed consent. After exclusion, a total of 550 patients who underwent TKA at either The Second Hospital of Hebei Medical University Department of Orthopedics or the Chongqing Medical University between January and March 2018 were enrolled in this study.

\subsection{Randomization}

In this study, block randomization was performed, with a randomized allocation of 1:1. Randomization was performed using an electronic data capture (EDC) system. The random list was concealed and stored on EDC servers with the randomization allocation made visible; the third party placed the random list into sequentially-coded sealed and opaque envelopes. After the sample size was determined, the third party opened the envelopes in order and randomly assigned the participants to the appropriate groups (phase exercise rehabilitation group or control group). The data analysts and participants were blinded during this study.

\subsection{Phase exercise rehabilitation program (rehabilitation group)}

The phase exercise rehabilitation program mainly included lower limb static training, lower limb dynamic training, lower limb flexibility training, and gluteus muscle and core strength training, all of which were performed for 45 minutes per day (Table 1). Every patient in the exercise rehabilitation group underwent the same rehabilitation therapy for four weeks that was prescribed by the same doctors. The patients were required to undergo follow-up evaluations at 1, 2, 4, 5, and 6 months during rehabilitation, and the effects of the rehabilitation program were assessed. Nonsteroidal antiinflammatory drugs or other analgesics were not used during the exercise rehabilitation program; if participants were in moderate pain (Visual Analogue Scale [VAS] $\geq 3$ ), they could use analgesics as a temporary measure and were asked to record their pain intensity on a 100-mm VAS. 
Table 1. Rehabilitation program for patients following total knee arthroplasty

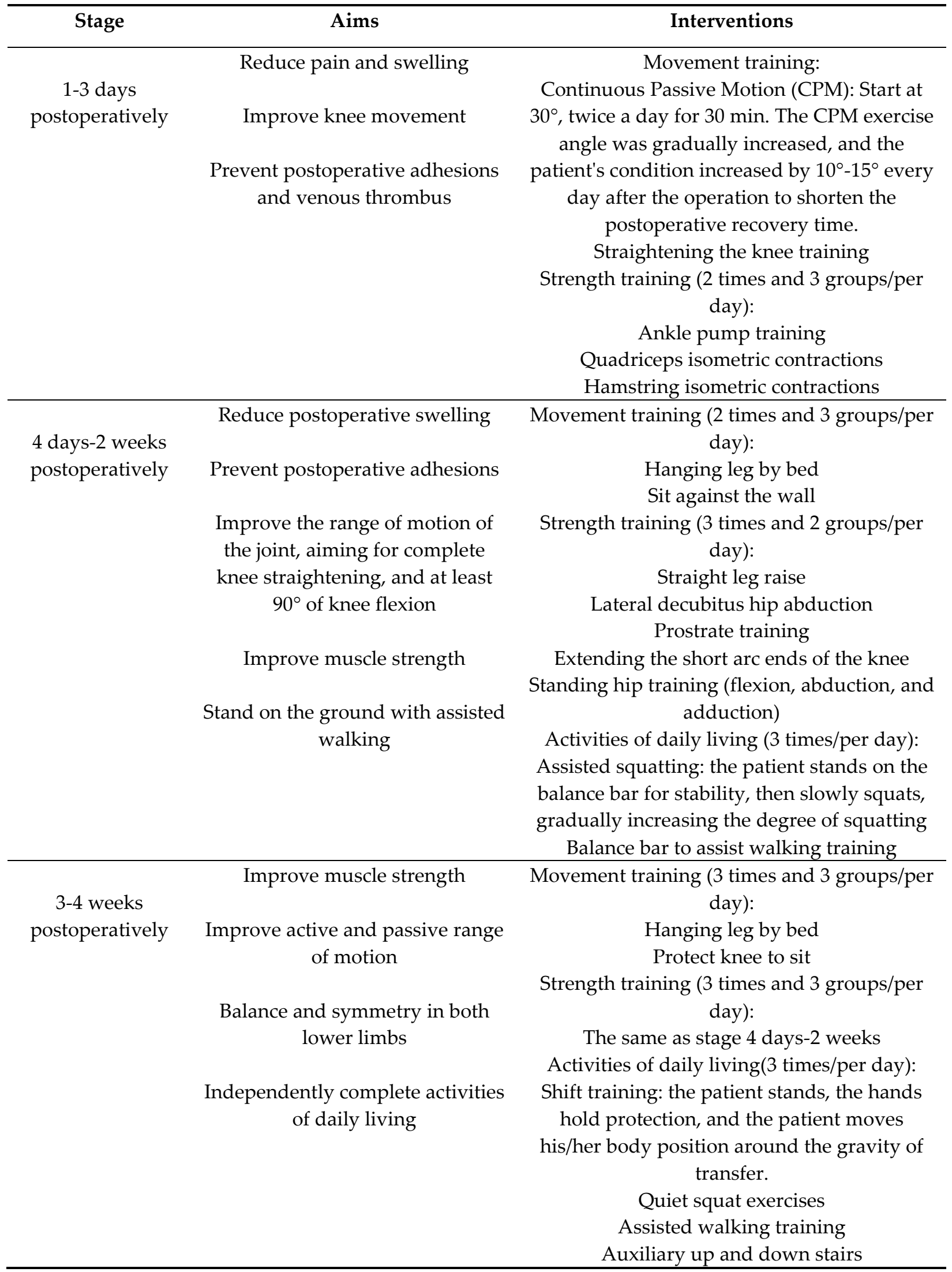


Patients in the control group received condition postoperative care that included muscle strength training, ice treatment, and transcutaneous electrical neural stimulation. Condition postoperative care was performed for 20 minutes per day for eight weeks. The patients were also required to undergo follow-up evaluations at 1, 2, 4, 5, and 6 months during rehabilitation, and the effects of the rehabilitation program were assessed.

\subsection{Assessment outcomes}

The outcomes and endpoints were reliant on the improvement of symptoms and pain, knee function, range of knee motion, and the quality of life of the patients. All outcomes were assessed for all patients before and after treatment at their attending medical center.

\subsection{Primary outcomes}

The primary outcomes were the clinical effectiveness of the treatment, changes in pain intensity, and improved symptoms. These outcomes were assessed using the 100-mm VAS and the Western Ontario and McMaster Universities Arthritis Index (WOMAC).

\subsection{Secondary outcomes}

The secondary outcomes were the range of motion of the knee and changes in the quality of life. The quality of life was measured using the Short-Form 36 (SF-36) questionnaire.

\subsection{Sample size calculation}

This study included a parallel-group design. To estimate the number of eligible participants, we used the results of unpublished data from a pilot study with an assumed level of significance of $\alpha=0.05$ (bilateral), a power of 0.75 , and type II error $(\beta)$ set to 0.2 . According to the unpublished data from the pilot study, the WOMAC score was the primary outcome for patients after TKA, with an effect size of 0.55 based on $65 \%$ compliance. In this study, the sample size was calculated using PASS software version 11.0 (NCSS, LLC, UT, USA), which resulted in a sample size of 167 participants in the control group and 334 participants in the intervention group. Considering a 10\% drop-out rate, the appropriate sample size for this study was 183 participants in the control group and 367 participants in the rehabilitation group, with a total of 550 participants. The flow chart for patient selection and randomization is shown in Figure 1. 


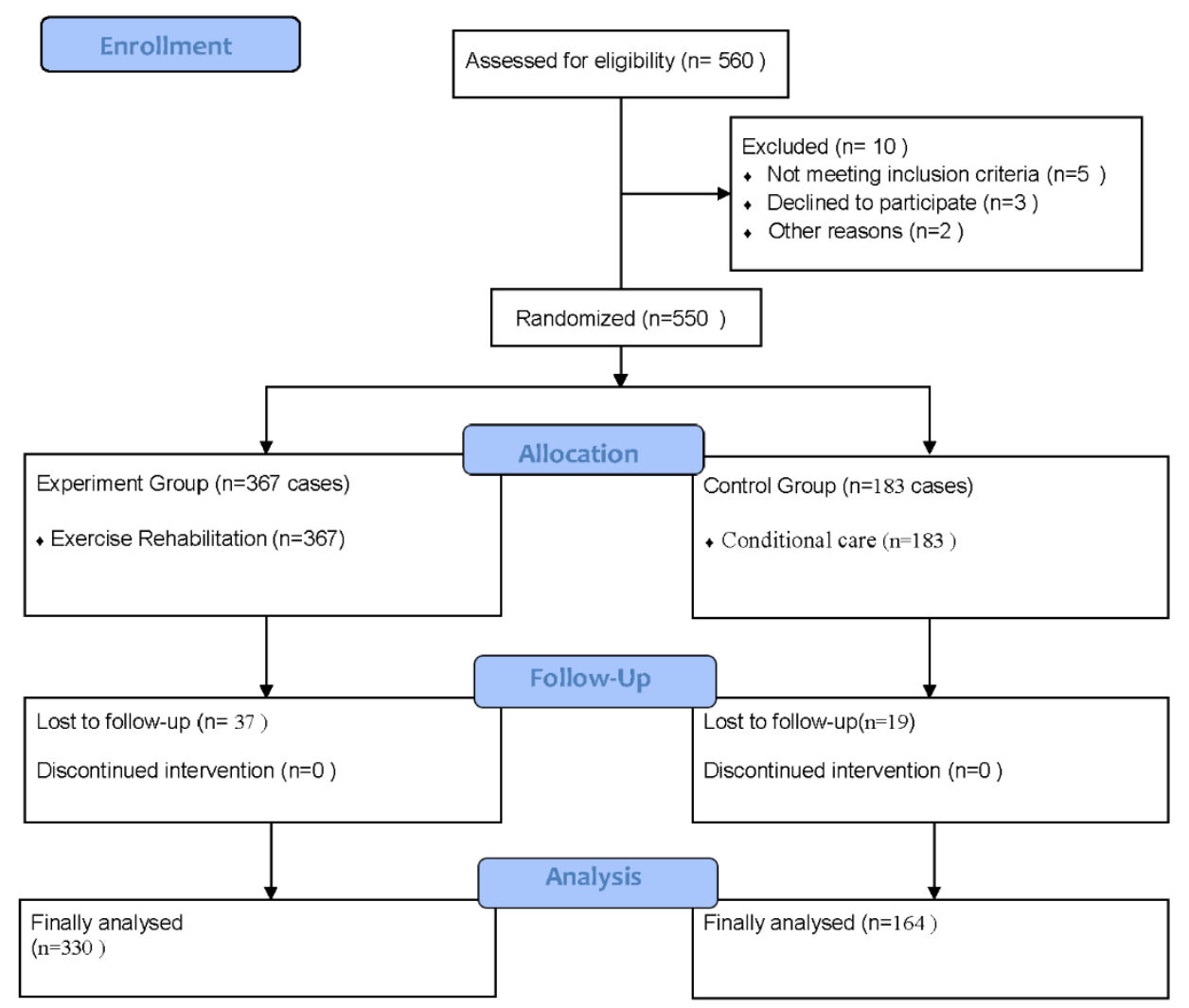

Figure 1. Flow diagram of patient selection and randomization for this controlled trial. In this study, we assessed the eligibility of 550 cases for enrollment, following exclusions, 494 cases were finally included for analysis. The experimental group included 330 cases, and the control group included 164 cases.

\subsection{Statistical analyses}

Comparisons of the primary and secondary outcomes between the groups at baseline and during the six months of follow-up were analyzed using a mixed model with patients as a random effect. All measurements (body mass index [BMI], VAS score, WOMAC score, SF-36 score, and range of motion of the knee) were analyzed using the paired sample $t$-test. All data pertaining to and adverse events of the two groups were analyzed using the Chi-squared test which was also used to compare the differences between male and female patients. A $p$-value of $<0.05$ was considered statistically significant. SPSS version 24.0 (IBM Corp, Inc., Chicago, IL, USA) was used for all statistical analyses.

\section{Results}

A total of 494 participants were included in this randomized controlled trial because 56 patients $(10.18 \%)$ were lost to follow-up. The mean age was $63.64 \pm 4.22$ years in the rehabilitation group and $64.32 \pm 5.10$ years in the control group. The average length of follow-up was $6 \pm 3.40$ months. There were no significant differences in age, sex, BMI, WOMAC score, VAS score, SF-36 score, and range of motion between the rehabilitation and control groups at baseline ( $p>0.05$; Table 2$)$. 
Table 2. Comparison of baseline characteristics between the two groups

\begin{tabular}{cccc}
\hline Characteristics & $\begin{array}{c}\text { Rehabilitation } \\
\text { group } \\
(\mathbf{M e a n} \pm \text { SD }) \\
(\mathbf{n}=\mathbf{3 3 0})\end{array}$ & $\begin{array}{c}\text { Control group } \\
(\text { Mean } \pm \text { SD) } \\
(\mathbf{n}=\mathbf{1 6 4})\end{array}$ & $p$ value \\
\hline Age (years) & $63.64 \pm 4.22$ & $64.32 \pm 5.10$ & 0.41 \\
Sex (Male:Female) & $146: 184$ & $103: 61$ & 0.83 \\
BMI & $26.26 \pm 2.90$ & $26.18 \pm 3.35$ & 0.32 \\
WOMAC Score & $113.56 \pm 2.90$ & $112.94 \pm 2.95$ & 0.28 \\
VAS Score & $8.03 \pm 2.17$ & $8.12 \pm 2.23$ & 0.17 \\
SF-36 Score & $78.07 \pm 3.21$ & $77.85 \pm 1.88$ & 0.78 \\
ROM & $114.54 \pm 3.10$ & $113.11 \pm 1.23$ & 0.51
\end{tabular}

SD: standard deviation, BMI: body mass index, WOMAC: Western Ontario and McMaster Universities Osteoarthritis Index, VAS: visual analogue scale, SF-36: Short-Form 36, ROM: range of motion

We found significant differences between the two groups regarding the functional and pain outcomes of the knee. The average WOMAC score was $84.40 \pm 15.20$ in the rehabilitation group and $108.30 \pm 3.90$ in the control group $(p=0.009)$. The mean VAS score was $2.54 \pm 0.16$ in the rehabilitation group and $2.87 \pm 0.31$ in the control group ( $p=0.024$ ). We also found significant improvements for the range of motion and quality of life in the rehabilitation group.

Table 3. Comparison of patient outcomes between the two groups

\begin{tabular}{cccc}
\hline Outcome mean \pm SD & Rehabilitation group & Control group & p value \\
\hline WOMAC Score & $84.40 \pm 15.20$ & $108.30 \pm 3.90$ & $0.009^{* *}$ \\
VAS Score & $2.54 \pm 0.16$ & $2.87 \pm 0.31$ & $0.024^{*}$ \\
SF-36 Score & $105.40 \pm 21.50$ & $83.7 \pm 5.01$ & $0.043^{*}$ \\
ROM & $125.02 \pm 6.20$ & $116.40 \pm 1.40$ & $0.017^{*}$ \\
\hline
\end{tabular}

SD: standard deviation, WOMAC: Western Ontario and McMaster Universities Osteoarthritis Index, VAS: visual analogue scale, SF-36: Short-Form 36, ROM: range of motion, ${ }^{*} p<0.05,{ }^{* *} p<0.01$

\section{Discussion}

Our study findings suggest that the phase rehabilitation program could be more effective for improving pain, knee function, and quality of life in patients after TKA than condition postoperative care.

In general, TKA is the standard treatment for patients with end-stage knee osteoarthritis. By 2009, the number of patients undergoing TKA annually had increased to 1.2 per 1000 patients [10], and the number of patients undergoing TKA continues to increase. As many patients who undergo TKA experience decreased muscle strength, especially in the quadriceps and hamstring muscles, postoperative rehabilitation is becoming increasingly popular among patients with knee osteoarthritis [11]. Several studies have shown lower extremity muscle weakness and functional deficits in patients who have undergone TKA compared with age-matched control subjects who have not undergone TKA [12,13]. Muscle inhibition, which is characterized by decreased muscle strength, is the main cause of functional limitations in patients who undergo TKA, and some studies suggest that quadriceps strength is a strong predictor of functional performance following TKA. This may be due to the association between decreased quadriceps strength and decreased balance and proprioception [14,15]. Low muscle strength increases the risk of falling and may lead to a loss of independence, while high muscle strength in the lower limbs is associated with a lower risk of 
mortality [16,17]. Major clinical trials and guidelines suggest that physiotherapy functional exercises after patient discharge result in short-term benefits, such as improved daily living activities, reduced pain, increased joint proprioception, and increased postural control/knee stability, after primary elective TKA $[18,19,20]$. However, the effectiveness of exercise rehabilitation for patients who have undergone TKA remains unclear; that is, most systematic reviews and meta-analyses demonstrate a lack of high-quality evidence, while most randomized clinical trials include a small sample size [21,22].

Recently, a systematic review of controlled trials demonstrated that the short- and long-term outcomes after TKA may be related to the type and intensity of postoperative rehabilitation that the patients receive [24]. However, determining the types and intensities of postoperative rehabilitation is still controversial. The Cochrane review suggests that continuous passive motions do not have clinically important effects on the active knee flexion range of motion, pain, function, or quality of life; however, it may reduce the risk of manipulation under anesthesia and the prevalence of adverse events [23]. In this study, the phase exercise rehabilitation program included a warm-up period, specific strengthening exercises, functional exercises, endurance exercises, and a cool-down period. The program focused on restoring the range of motion of the knee and knee muscle strength, the development of functional independence, establishing proprioception exercises to encourage walking without a limp, and improving the ability of patients to perform recreational activities. The primary purpose of this rehabilitation program was to maximize the early range of motions, improve strength, reduce pain, and normalize gait mechanics in patients with knee osteoarthritis.

In this rehabilitation project, we focused on decreasing knee pain and swelling, remodeling and improving knee mobility under painless conditions, improving muscle strength during different types of contractions, restoring normal gait patterns, and neuromuscular coordination. Previous clinical trials have only studied the effectiveness of muscle strength training for patients after TKA $[11,15]$; these studies may have an increased number of patients lost to follow-up. Furthermore, because simple exercise styles could cause the patients to become exhausted more quickly, the training was not as effective as expected.

In this trial, the exercises focused on balance and proprioception and were combined with exercises to control pain and chronic inflammation; these exercises aimed to restore postoperative knee joint and soft tissue tension and enhance mechanoreceptor responses in the knee and muscles, thereby increasing joint motion and position sensation. We demonstrated that our rehabilitation project was more effective at improving lower limb movement and control, relieving pain, improving mobility, and reducing falls than condition postoperative care.

This study had some limitations that should be addressed. First, we did not measure the success of assessor blinding, the primary and secondary outcomes were self-reported, and the follow-up time was relatively short. Thus, the outcome measurements are not subjected to possible assessor or reporting biases. Future studies should focus on the long-term follow-up of patients after TKA and increase functional and serological testing of patients which could be used to better elucidate potential mechanisms underlying phase exercise rehabilitation programs and their effects on patients after TKA. Low- and moderate-quality evidence has demonstrated that clinic-based or inpatient programs were not superior for patients after TKA compared to home-based programs. However, home-based rehabilitation programs did provide greater mobility than clinic-based programs [25]. Future studies could focus on increasing patient compliance with home-based rehabilitation programs and improving the functional and quality of life outcomes in patients who have undergone TKA.

In conclusion, this study suggests that phase rehabilitation programs could be more effective at relieving pain, improving knee function, and increasing the quality of life for patients after TKA, compared with condition postoperative care.

Author Contributions: Study protocol design: Jing, Yang, Bai; Drafting of the manuscript: Jing, Lee, Yang; Critical revision of the manuscript: Yang, Lee, Gong; Data collection and analysis: Jing, Yang, Gong; Obtain funding: Jing, Bai, Gong. 
Funding: This work was funded by the Natural Science Foundation of Hebei Province, grant number A2019001343, and the Hebei Provincial Department of Science and Technology.

Conflicts of Interest: No authors declare conflicts of interest.

\section{References}

1. Melvin, J.S.; Stryker, L.S.; Sierra, R.J. Tranexamic acid in hip and knee arthroplasty. J. Am. Acad. Orthop. Surg. 2015, 23, 732-740; DOI:10.5435/JAAOS-D-14-00223.

2. Tang, X.; Wang, S.; Zhan, S.; Niu, J.; Tao, K.; Zhang, Y.; Lin, J. The prevalence of symptomatic knee osteoarthritis in China: Results From the China Health and Retirement Longitudinal Study. Arthritis Rheumatol. 2016, 68, 648-653; DOI:10.1002/art.39465.

3. Pozzi, F.; Snyder-Mackler, L.; Zeni, J. Physical exercise after knee arthroplasty: A systematic review of controlled trials. Eur. J. Phys. Rehabil. Med. 2013, 49, 877-892.

4. Buhagiar, M.A.; Naylor, J.M.; Harris, I.A.; Xuan, W.; Adie, S.; Lewin, A. Assessment of outcomes of inpatient or clinic-based vs home-based rehabilitation after total knee arthroplasty: A systematic review and meta-analysis. JAMA Netw. Open. 2019, 2, e192810; DOI:10.1001/jamanetworkopen.2019.2810.

5. Bade, M.J.; Struessel, T.; Dayton, M.; Foran, J.; Kim, R.H.; Miner, T.; Wolfe, P.; Kohrt, W.M.; Dennis, D.; Stevens-Lapsley, J.E. Early high-intensity versus low-intensity rehabilitation after total knee arthroplasty: A randomized controlled trial. Arthritis Care Res. (Hoboken). 2017, 69, 1360-1368; DOI:10.1002/acr.23139.

6. Moffet, H.; Tousignant, M.; Nadeau, S.; Mérette, C.; Boissy, P.; Corriveau, H.; Marquis, F.; Cabana, F.; Ranger, P.; Belzile, É.L.; Dimentberg, R. In-home telerehabilitation compared with face-to-face rehabilitation after total knee arthroplasty: A noninferiority randomized controlled trial. J. Bone Joint Surg. Am. 2015, 97, 1129-1141; DOI:10.2106/JBJS.N.01066.

7. Gill, S.D.; McBurney, H. Does exercise reduce pain and improve physical function before hip or knee replacement surgery: A systematic review and meta-analysis of randomized controlled trials. Arch. Phys. Med. Rehabil. 2013, 94, 164-176; DOI:10.1016/j.apmr.2012.08.211.

8. Schache, M.B.; McClelland, J.A.; Webster, K.E. Lower limb strength following total knee arthroplasty: A systematic review. Knee. 2014, 21, 12-20; DOI:10.1016/j.knee.2013.08.002.

9. Tilbury, C.; Schaasberg, W.; Plevier, J,W.; Fiocco, M.; Nelissen, R.G.; Vliet Vlieland, T.P. Return to work after total hip and knee arthroplasty: a systematic review. Rheumatology (Oxford). 2014, 53, 512-525; DOI:10.1093/rheumatology/ket389.

10. Stevens-Lapsley, J.E.; Balter, J.E.; Kohrt, W.M.; Eckhoff, D.G. Quadriceps and hamstrings muscle dysfunction after total knee arthroplasty. Clin. Orthop. Relat. Res. 2010, 468, 2460-2468; DOI:10.1007/s11999009-1219-6.

11. Mistry, J.B.; Elmallah, R.D.; Bhave, A.; Chughtai, M.; Cherian, J.J.; McGinn, T.; Harwin, S.F.; Mont, M.A. Rehabilitative guidelines after total knee arthroplasty: A review. J. Knee Surg. 2016, 29, 201-207; DOI:10.1055/s-0036-1579670.

12. Núñez, M.; Lozano, L.; Núñez, E.; Segur, J.M.; Sastre, S.; Maculé, F.; Ortega, R.; Suso, S. Total knee replacement and health-related quality of life: factors influencing long-term outcomes. Arthritis Rheum. 2009, 61, 1062-1069; DOI:10.1002/art.24644.

13. Yoshida, Y.; Mizner, R.L.; Ramsey, D.K.; Snyder-Mackler, L. Examining outcomes from total knee arthroplasty and the relationship between quadriceps strength and knee function over time. Clin. Biomech. (Bristol, Avon). 2008, 23, 320-328; DOI:10.1016/j.clinbiomech.2007.10.008.

14. Ardali, G. A daily adjustable progressive resistance exercise protocol and functional training to increase quadriceps muscle strength and functional performance in an elderly homebound patient following a total knee arthroplasty. Physiother. Theory Pract. 2014, 30, 287-297; DOI:10.3109/09593985.2013.868064.

15. Altman, R.D.; Bedi, A.; Karlsson, J.; Sancheti, P.; Schemitsch, E. Product differences in intra-articular hyaluronic acids for oteoarthritis of the knee. Am. J. Sports Med. 2016, 44, 2158-2165; DOI:10.1177/0363546515609599.

16. Ethgen, O.; Bruyère, O.; Richy, F.; Dardennes, C.; Reginster, J.Y. Health-related quality of life in total hip and total knee arthroplasty. A qualitative and systematic review of the literature. J. Bone Joint Surg. Am. 2004, 86, 963-974; DOI:10.2106/00004623-200405000-00012.

17. Villadsen, A.; Overgaard, S.; Holsgaard-Larsen, A.; Christensen, R.; Roos, E.M. Postoperative effects of neuromuscular exercise prior to hip or knee arthroplasty: A randomised controlled trial. Ann. Rheum. Dis. 2014, 73, 1130-1137; DOI:10.1136/annrheumdis-2012-203135. 
18. Dziedzic, K.S.; Healey, E.L.; Porcheret, M.; Afolabi, E.K.; Lewis, M.; Morden, A.; Jinks, C.; McHugh, G.A.; Ryan, S.; Finney, A.; Main, C.; Edwards, J.J.; Paskins, Z.; Pushpa-Rajah, A.; Hay, E.M. Implementing core NICE guidelines for osteoarthritis in primary care with a model consultation (MOSAICS): A cluster randomised controlled trial. Osteoarthritis Cartilage. 2018, 26, 43-53; DOI:10.1016/j.joca.2017.09.010.

19. Aresti, N.; Kassam, J.; Bartlett, D.; Kutty, S. Primary care management of postoperative shoulder, hip, and knee arthroplasty. BMJ. 2017, 359, j4431; DOI:10.1136/bmj.j4431.

20. Minns Lowe, C.J.; Barker, K.L.; Dewey, M.; Sackley, C.M. Effectiveness of physiotherapy exercise after knee arthroplasty for osteoarthritis: Systematic review and meta-analysis of randomised controlled trials. BMJ. 2007, 335, 812; DOI:10.1136/bmj.39311.460093.BE.

21. Cushnaghan, J.; Bennett, J.; Reading, I.; Croft, P.; Byng, P.; Cox, K.; Dieppe, P.; Coggon, D.; Cooper, C. Longterm outcome following total knee arthroplasty: A controlled longitudinal study. Ann. Rheum. Dis. 2009, 68, 642-647; DOI:10.1136/ard.2008.093229.

22. Liao, C.D.; Huang, Y.C.; Lin, L.F.; Chiu, Y.S.; Tsai, J.C.; Chen, C.L.; Liou, T.H. Continuous passive motion and its effects on knee flexion after total knee arthroplasty in patients with knee osteoarthritis. Knee Surg. Sports Traumatol. Arthrosc. 2016, 24, 2578-2586; DOI:10.1007/s00167-015-3754-x.

23. Fortin, P.R.; Penrod, J.R.; Clarke, A.E.; St-Pierre, Y.; Joseph, L.; Bélisle, P.; Liang, M.H.; Ferland, D.; Phillips, C.B.; Mahomed, N.; Tanzer, M.; Sledge, C.; Fossel, A.H.; Katz, J.N. Timing of total joint replacement affects clinical outcomes among patients with osteoarthritis of the hip or knee. Arthritis Rheum. 2002, 46, 3327-3330; DOI:10.1002/art.10631.

24. Husby, V.S.; Foss, O.A.; Husby, O.S.; Winther, S.B. Randomized controlled trial of maximal strength training vs. standard rehabilitation following total knee arthroplasty. Eur. J. Phys. Rehabil. Med. 2018, 54, 371-379; DOI:10.23736/S1973-9087.17.04712-8.

25. Kramer, J.F.; Speechley, M.; Bourne, R.; Rorabeck, C.; Vaz, M. Comparison of clinic- and home-based rehabilitation programs after total knee arthroplasty. Clin. Orthop. Relat. Res. 2003, 410, 225-234; DOI:10.1097/01.blo.0000063600.67412.11. 